\title{
The impact of host
}

\section{aggressiveness on sex allocation by the gregarious parasitoid wasp Cotesia glomerata (L.)}

\author{
Shingo Tanaka* \\ Center for Sustainability Science, Hokkaido University, \\ Sapporo 060-0809, Fapan \\ *tanaka-s@sgp.hokudai.ac.jp
}

Parasitoid wasps lay female eggs or a high proportion of female eggs in favourable host insects because female wasps require many more resources during their development. Many studies have tested the effects of host physiological status on the sex allocation of parasitoids, but few have attempted to test the effects of host behavioural traits. Cotesia glomerata is a gregarious parasitoid wasp that lays eggs in caterpillars of pierid butterflies. The brood sex ratio in C. glomerata females that attacked aggressive host caterpillars was compared with that in females that attacked less aggressive hosts. The male ratio was higher when $C$. glomerata attacked aggressive Pieris brassicae caterpillars than when it attacked less aggressive Pieris rapae crucivora caterpillars. However, when $C$. glomerata females were induced to oviposit in anaesthetized $P$. brassicae caterpillars, the male ratio in their offspring was significantly lower than when they attacked unanaesthetized caterpillars. C. glomerata was attacked by aggressive host caterpillars during oviposition bouts. It is likely that this aggressive host behaviour disturbed the fertilization process in ovipositing $C$. glomerata females. These results suggest that a behavioural defence by host caterpillars affects sex allocation in the parasitoid wasp $C$. glomerata.

Keywords: sex ratio; behavioural defence; behavioural constraints; oviposition behaviour; Cotesia glomerata (L.)

\section{INTRODUCTION}

The typical sex determination system of hymenopteran parasitoids is arrhenotokous haplodiploidy: fertilized eggs develop into females and unfertilized eggs develop into males. Therefore, female wasps are able to produce progenies of the preferred sex depending on various conditions associated with their hosts. For example, sex allocation strategies of some parasitoid wasps may vary with host size (Charnov et al. 1981), developmental stage (King 1990; Joyce et al. 2002) or species (Kochetova 1978; Wang \& Messing 2004). In any case, wasps tend to lay female eggs on preferred hosts in terms of developmental performance, because female reproductive success is increased more by qualitatively or quantitatively preferable

resources than male reproductive success (Jones 1982; van den Assem et al. 1989). However, the most desirable strategy may be blocked by many kinds of disturbances, including host defences. Oviposition behaviours of some parasitoids, which lay eggs without temporary or permanent paralysis of their hosts, would probably be affected by defensive attacks by their hosts. No studies have examined the impacts of behavioural defence by hosts on sex allocation by parasitoid wasps.

Cotesia glomerata (L.) is a gregarious koinobiont wasp that parasitizes caterpillars of pierid butterflies. This wasp lays approximately 20 eggs in a first to third instar caterpillar without temporary paralysis. The sex allocation of $C$. glomerata suggests partial local mate competition (Gu \& Dorn 2003), and, consequently, the wasp produces female-biased broods (Brodeur et al. 1998; Tagawa 2000; Harvey et al. 2004). In Japan, C. glomerata parasitizes mainly Pieris rapae crucivora Boisduval, but in northern Japan, it has also used Pieris brassicae (L.) since the invasion of this butterfly approximately the mid-1990s (Tanaka et al. 2007). For C. glomerata, the physiological qualities of the two host species are almost equal, provided that progeny derived from a single clutch develops in a host caterpillar. However, these host species differ completely in their behavioural traits (Brodeur et al. 1996). P. rapae crucivora is solitary and benign, whereas $P$. brassicae is gregarious and aggressive. The difference in their behavioural traits possibly affects host estimation by $C$. glomerata. To eliminate possible adaptation to the aggressive defence of invasive P. brassicae, brood sex ratios in a Kyoto population, which has not experienced $P$. brassicae, were compared with those in a Sapporo population, which could potentially attack that species. Then, the brood sex ratios of $C$. glomerata females that attacked aggressive P. brassicae were compared with those of females that attacked anaesthetized $P$. brassicae to separate the effects of physiological characteristics from those of host aggressiveness. The results of the experiments confirmed the effects of host aggressiveness on sex ratios of $C$. glomerata.

\section{MATERIAL AND METHODS}

Cotesia glomerata and host butterflies were collected from cabbage fields in Sapporo and Kyoto. All collections in Sapporo were conducted in a field of the National Agricultural Research Center for the Hokkaido Region, with the permission of Dr J. Kaneko. Emerged wasps that originated from a single host caterpillar were reared in a test tube (diameter, $1.8 \mathrm{~cm}$; length, $18 \mathrm{~cm}$ ) and allowed to inbreed. The following experiments were conducted from 09.00 to 18.00 with 4 - to 6 -day-old naive female wasps. The insects were reared and the experiments conducted in the laboratory at $25 \pm 2{ }^{\circ} \mathrm{C}$ under $16: 8$ light:dark conditions.

So that the sizes of the host caterpillars would be comparable, late-stage first instar $P$. rapae and mid-stage first instar $P$. brassicae caterpillars were used for the experiments. First, the host species was evaluated as a factor affecting sex ratios. One caterpillar from each of the two species was given to a female wasp, and the wasp allowed to oviposit once. The parasitized caterpillar was retrieved and reared on cabbage leaves in plastic cups $(12 \mathrm{~cm}$ in diameter by $5 \mathrm{~cm}$ deep) to produce adults of the next generation. One day after their emergence, the offspring were euthanized in a freezer and the numbers of wasp cocoons, emerged individuals and male wasps were counted. Sample sizes were 72 (Kyoto $\times$ P. brassicae), 69 (Kyoto $\times$ P. rapae), 70 (Sapporo $\times$ P. brassicae) and 44 (Sapporo $\times$ P. rapae). Second, the effect of host aggressiveness was evaluated by using $P$. brassicae caterpillars. Each $C$. glomerata female was provided with either a caterpillar anaesthetized by carbon dioxide or one that was unanaesthetized. The oviposition trials were 
Table 1. Test results for the effects of population and host species on the sex ratio, with the number of wasps per cocoon cluster as a covariate.

\begin{tabular}{llrl}
\hline variables & d.f. & likelihood ratio $\chi^{2}$ & $p$-value \\
\hline populations & 1 & 182.3731 & $<0.0001$ \\
hosts & 1 & 23.5642 & $<0.0001$ \\
number of wasps & 1 & 31.0904 & $<0.0001$ \\
\hline
\end{tabular}

conducted as described, except that second instar caterpillars were provided as hosts. In this experiment, 38 anaesthetized and 28 unanaesthetized caterpillars were used.

The data were analysed statistically by logistic regression to explain the variation in sex allocation. In the statistical model, the populations and host species and the interactions between them were treated as independent variables, and the number of emerged wasps per cocoon cluster of $C$. glomerata as a covariate. In the first model, all the interactions between the covariate and the independent variables (population $\times$ host, population $\times$ number of wasps, host $\times$ number of wasps and population $\times$ host $\times$ number of wasps) were included. However, when the interactions were not significant, they were removed from the model, and analysis was performed again based on a new model. We also compared the brood size in different host species by using two-way analysis of variance. The wasp population, host species and the interactions between them were treated as independent variables in the statistical model. Statistical procedures were run using JMP 4.0.5 (SAS Institute Inc., Cary, NC, USA).

\section{RESULTS}

Logistic regression indicated that none of the interactions between the covariate and the independent variables was significant (population $\times$ host for d.f. $=1, p=0.9717$; population $\times$ number of wasps for d.f. $=1, p=0.3431$; host $\times$ number of wasps for d.f. $=1, p=0.1582$; and population $\times$ host $\times$ number of wasps for d.f. $=1, p=0.9639$ ) Thus, logistic regression was recalculated by removing the interactions from the model (table 1). The brood sex ratio (male ratio) of C. glomerata females that oviposited in P. brassicae was higher than that in females that oviposited in $P$ rapae (figure 1 ; table 1 ). In addition, the sex ratio differed between the two wasp populations. The male ratio in the Sapporo population was significantly higher than that in the Kyoto population irrespective of host species. Although the male ratio was high in small broods, the brood size neither differ significantly between the two host species $\left(F_{1,251}=1.2338, p=0.2677\right)$ nor between the two populations $\left(F_{1,251}=2.5536, p=0.1113\right)$.

The brood sex ratio (male ratio) in females that oviposited in unanaesthetized $P$. brassicae caterpillars was higher than the male ratio in females that oviposited in anaesthetized caterpillars (whole model, d.f. $=3, \chi^{2}=89.5411, p<0.0001$; figure 2 , table 2). Unanaesthetized $P$. brassicae caterpillars intensely bit or dragged ovipositing C. glomerata females when the wasp inserted its ovipositor into the caterpillars. The brood size did not differ significantly between unanaesthetized and anaesthetized P. brassicae caterpillars $\left(F_{1,65}=0.0042, p=0.9488\right.$, one-way ANOVA), although small broods slightly produced high proportion of male progeny, especially in unanaesthetized hosts.

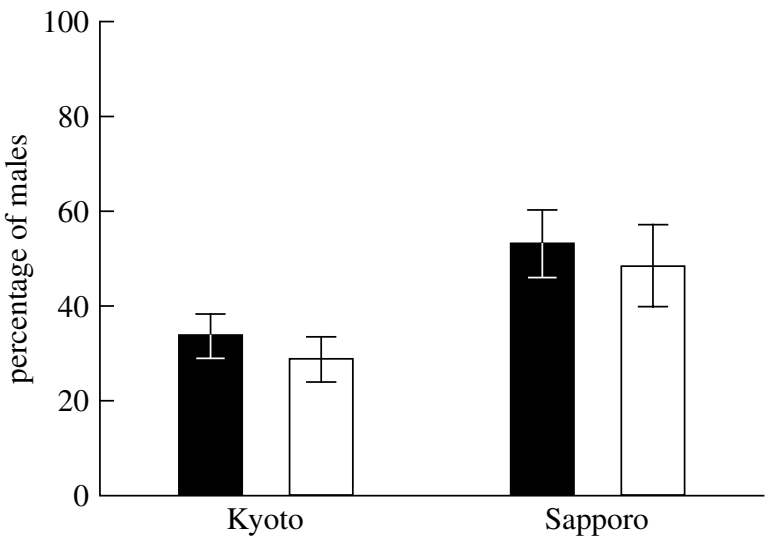

Figure 1. Brood sex ratios (male ratios) in two different populations of $C$. glomerata females that attacked two different host species: solid bars, P. brassicae; open bars, $P$. rapae. Male ratio means and $95 \%$ confidence intervals are shown.

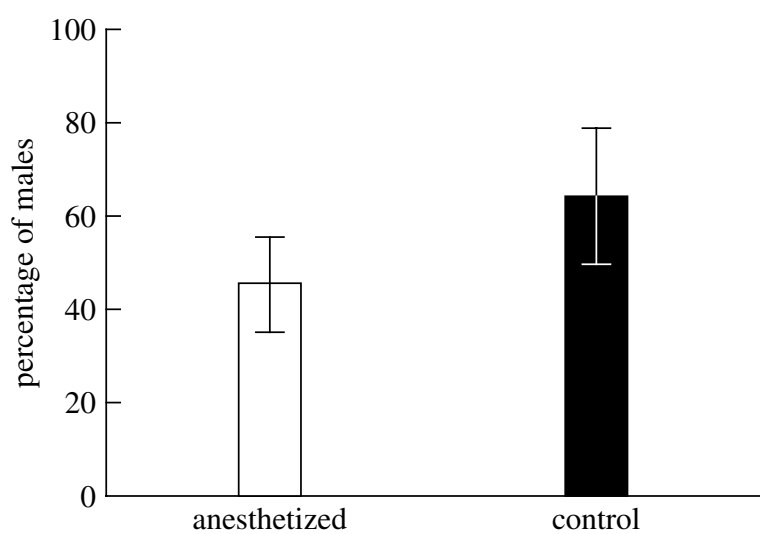

Figure 2. Brood sex ratios (male ratios) of $C$. glomerata females that attacked second instar caterpillars of $P$. brassicae that differed in their aggressiveness: solid bar, normal aggressive caterpillars; open bar, anaesthetized caterpillars. Male ratio means and $95 \%$ confidence intervals are shown.

Table 2. Test results for the effect of host condition (anaesthetized/unanaesthetized) on the sex ratio, with the number of wasps per cocoon cluster as a covariate.

\begin{tabular}{lllr}
\hline variables & d.f. & likelihood ratio $\chi^{2}$ & \multicolumn{1}{c}{$p$-value } \\
\hline condition of hosts & 1 & 45.4123 & $<0.0001$ \\
number of wasps & 1 & 40.3924 & $<0.0001$ \\
$\begin{array}{l}\text { condition } \times \text { number } \\
\quad 1\end{array}$ & 10.9951 & 0.0009 \\
\multicolumn{1}{c}{ of wasps } & & & \\
\hline
\end{tabular}

\section{DISCUSSION}

The results of the present study show that C. glomerata females alter the sex allocation of their brood depending on host species, and that the male ratio is higher when females oviposit in $P$. brassicae than when females oviposit in P. rapae. This difference in sex ratio cannot be explained by a difference between the size of clutches deposited in the two host species, but can be explained by the differences in belligerence of the host caterpillars, which would intensely affect oviposition behaviours of wasp females irrespective of their population of origin. This hypothesis was confirmed by the results of an 
experiment with anaesthetized and unanaesthetized hosts; the male ratio of the $C$. glomerata brood was higher in unanaesthetized aggressive caterpillar hosts than in anaesthetized caterpillar hosts. The host caterpillars used in this experiment differed only in their aggressiveness; the species and instars were the same.

When female wasps fertilize the eggs at the moment of oviposition, they suspend their motion for several seconds. The duration of motionless behaviour is significantly longer when the female wasps lay female eggs than when they lay male eggs (Yamada \& Kawamura 1999). Such a sex-biased difference in ovipositional behaviour has been reported from many wasp species (e.g. Cole 1981; Strand 1989; Ueno 1995). Female wasps occasionally fail to fertilize some eggs even while ovipositing into paralysed hosts (Yamada \& Kawamura 1999). If wasps are attacked by their hosts during oviposition, they may more often fail to fertilize eggs. Consequently, since $C$. glomerata females are more frequently attacked by $P$. brassicae caterpillars than by P. rapae caterpillars, such interference may lead to a higher male ratio in the brood deposited in P. brassicae caterpillars.

The high male ratio in aggressive caterpillars still may be explained from a viewpoint of ecological adaptation. One has to consider that only a decade has passed since Japanese $C$. glomerata began to attack invasive $P$. brassicae. In comparison, European C. glomerata would have achieved a counter adaptation against aggressive defence of $P$. brassicae caterpillars to ensure high fertilization success under partial LMC condition through a long evolutionary history. Although European C. glomerata exhibits high male ratios in brood oviposited in $P$. brassicae (Brodeur et al. 1998; Harvey 2000), it may be due to group defence by gregarious $P$. brassicae caterpillars. Early instar caterpillars of the butterfly form an aggregate and some of them join in an attack against parasitizing C. glomerata. Therefore, it is possible that attack by a single host caterpillar affects sex allocation by specialist parasitoids only when their adaptations to an invasive host have not been accomplished. In time, however, Japanese $C$. glomerata may attain the high fertilization success in $P$. brassicae.

The author would like to thank Dr N. Ohsaki, Kyoto University, Dr S. Akimoto, Hokkaido University and Dr J. Tagawa, Okayama University of Science, for their helpful advice and encouragement.

Brodeur, J., Geervliet, J. B. F. \& Vet, L. E. M. 1996 The role of host species, age and defensive behaviour on ovipositional decisions in a solitary specialist and gregarious generalist parasitoid (Cotesia species). Entomol. Exp. Appl. 81, 125-132. (doi:10.1007/BF00192137)

Brodeur, J., Geervliet, J. B. F. \& Vet, L. E. M. 1998 Effects of Pieris host species on life history parameters in a solitary specialist and gregarious generalist parasitoid (Cotesia species). Entomol. Exp. Appl. 86, 145-152. (doi:10.1023/A:1003199515445)

Charnov, E. L., Los-den Hartogh, R. L., Jones, W. T. \& van den Assem, J. 1981 Sex ratio evolution in a variable environment. Nature 289, 27-33. (doi:10.1038/ 289027a0)

Cole, L. R. 1981 A visible sign of a fertilization action during oviposition by an ichneumonid wasp, Itoplectis maculator. Anim. Behav. 29, 299-300. (doi:10.1016/ S0003-3472(81)80178-9)

Gu, H. \& Dorn, S. 2003 Mating system and sex allocation in the gregarious parasitoid Cotesia glomerata. Anim. Behav. 66, 259-264. (doi:10.1006/anbe.2003.2185)

Harvey, J. A. 2000 Dynamic effects of parasitism by an endoparasitoid wasp on the development of two host species: implications for host quality and parasitoid fitness. Ecol. Entomol. 25, 267-278. (doi:10.1046/j.13652311.2000.00265.x)

Harvey, J. A., Witjes, L. M. A. \& Wagenaar, R. 2004 Development of hyperparasitoid wasp Lysibia nana (Hymenoptera: Ichneumonidae) in a multitrophic framework. Environ. Entomol. 33, 1488-1496.

Jones, W. T. 1982 Sex ratio and host size in a parasitic wasp. Behav. Ecol. Sociobiol. 10, 207-210. (doi:10.1007/ BF00299686)

Joyce, A. L., Millar, J. G., Paine, T. D. \& Hanks, L. M. 2002 The effect of host size on the sex ratio of Syngaster lepidus, a parasitoid of Eucalyptus longhorned borers (Phoracantha spp.). Biol. Control 24, 207-213. (doi:10. 1016/S1049-9644(02)00031-2)

King, B. H. 1990 Sex ratio manipulation by the parasitoid wasp Spalangia cameroni in response to host age: a test of the host-size model. Evol. Ecol. 4, 149-156. (doi:10. 1007/BF02270912)

Kochetova, N. I. 1978 Factors determining the sex ratio in some entomophagous hymenoptera. Entomol. Rev. 57, $1-5$.

Strand, M. R. 1989 Oviposition behavior and progeny allocation of the polyembryonic wasp Copidosoma floridarum (Hymenoptera: Encyrtidae). F. Insect Behav. 2, 355-369. (doi:10.1007/BF01068061)

Tagawa, J. 2000 Sex allocation and clutch size in the gregarious larval endoparasitoid wasp, Cotesia glomerata. Entomol. Exp. Appl. 97, 193-202. (doi:10.1023/ A:1004007802543)

Tanaka, S., Nishida, T. \& Ohsaki, N. 2007 Sequential rapid adaptation of indigenous parasitoid wasps to the invasive butterfly Pieris brassicae. Evolution 61, 1791-1802. (doi:10.1111/j.1558-5646.2007.00165.x)

Ueno, T. 1995 Abdominal tip movements during oviposition by two parasitoids (Hymenoptera: Ichneumonidae) as an index of predicting the sex of depositing eggs. Appl. Entomol. Zool. 30, 588-590.

van den Assem, J., van Iersal, J. A. \& Los-den Hartogh, R. L. 1989 Is being large more important for female than male parasitic wasps? Behaviour 108, 160-195. (doi:10.1163/156853989X00114)

Wang, X. G. \& Messing, R. H. 2004 Fitness consequences of body-size-dependent host species selection in a generalist ectoparasitoid. Behav. Ecol. Sociobiol. 56, 513-522. (doi:10.1007/s00265-004-0829-y)

Yamada, Y. Y. \& Kawamura, M. 1999 Sex identification of eggs of a dryinid parasitoid, Haplogonatopus atratus, based on oviposition behaviour. Entomol. Exp. Appl. 93, 321-324. (doi:10.1023/A:1003980621839) 